\title{
Sedimentation behaviour study by three optical methods - granulometric and electrophoresis measurements, dispersion optical analyser
}

\author{
N. Azema* \\ Centre des Matériaux de Grande Diffusion, Ecole des Mines, 6 avenue de Clavières, 30319 Alès, Cédex, France
}

\begin{abstract}
Taking into account the importance of characterisation of stability of dispersed systems in both fundamental research and industrial technology, three optical techniques, were used and compared to study sedimentation behaviours of mineral suspensions.

The agglomeration and dispersion phenomena of mineral suspensions in water and ethanol media, has been studied by a granulometric and sedimentation approach (electrophoresis mobility, particle size distribution, clarifying speed, sediment formation).

So, this experimental work has perfectly shown the necessity to achieve a complementary approach by these three optical methods to completely investigate the sedimentation behaviours.
\end{abstract}

Keywords: Suspension stability; Sedimentation; Dispersion; Agglomeration; Characterisation; Optical methods

\section{Introduction}

In various application areas, many industrial products are dispersed systems, in particular, suspensions. Their stability or instability represent a significant property for controlling industrial processes and their environmental impact, product quality and are strongly affected by polarity of liquid media e.g. water, ethanol [1,2], which are commonly used.

The aim of the present work is to compare three optical methods (laser diffraction size analysis, and electrophoresis measurement, and concentrated dispersion analyser) to study the sedimentation behaviour of mineral suspensions and the polarity of liquid medium influence.

The dispersed systems studied are, in particular, used to treat $a$ metal surface during the braze process and require an application on the metal surface in $a$ stabilised suspension state. The solid phase is a mixing of two micron-sized powder of aluminium fluoride and aluminium hydroxide [3]. On the other hand, different polarities of liquid medium are obtained by mixtures of water and ethanol. We can notice that the industrial composition of a liquid medium was not optimised until this day to require a stabilised suspension.

\footnotetext{
* Tel.: +33 4667853 56; fax: +3346678 5365 .
}

E-mail address: nathalie.azema@ema.fr.
The sedimentation behaviour [4,5] is determined by particle characteristics such as size, particle-particle interaction, density of particles which mainly depends on granular, physicochemical or hydrodynamic conditions, or weight concentration $[6,7]$. However, in the micronic size range, the nature of the solid/liquid interface plays an important role in the modification of particle size distribution because of common phenomena such as dispersion, agglomeration, aggregation, or even dissolution. So, modifications of polarity of liquid medium may involve variation of potential surface which, in turn, may influence particle size distribution and sedimentation behaviours.

Then, the electrophoretic mobility [8] and the particle size distribution [9] were analysed respectively by electrophoresis and by laser granulometric measurements according to the liquid phase composition. Experimental relationships between both characteristics and the suspension stability were studied using a new dispersion analyser [10-12].

\section{Materials and methods}

\subsection{Laser granulometry and electrophoresis measurements}

The measurements of particle size distribution and electrophoretic mobility were performed using respectively a LS230 
laser granulometer and a DELSA 440SX electrophoresis measurement (Beckman-Coulter).

In the laser diffraction technology, the particle size distribution is determined on the basis of the scattering of monochromatic light with a wavelength of $750 \mathrm{~nm}$ transmitted through the suspension. The measurement of particle's diffraction pattern is characteristic of particle size [13]. The Fraunhofer optical model computes the flux pattern into a particle size distribution in the range of 0.04 to $2000 \mu \mathrm{m}$ of particle diameters. Each analysis is minimum reproduced twice.

The second method, namely Electrophoretic Light Scattering (ELS) is a technique that combines two technologies: electrophoresis and laser Doppler velocimetry [14]. The ELS system also uses laser light scattering. The particles move at constant velocity due to an applied electric field. They scatter the laser light with a shift of frequency compared to frequency of the incident light. This frequency shift is called a Doppler shift and is proportional to the particle speed. For each analysis with this apparatus, four measurements are realised simultaneously. The measurement of ionic conductivity is possible too.

\subsection{Optical analyser}

A recent optical analyser was used to study suspension behaviour and to determine sedimentation speed (Turbiscan MA 2000). This instrument detects and measures modifications of concentrated and opaque suspensions. The Turbiscan carries out step-by-step vertical scanning of the whole sample by a pulsed near-infrared light source $(\lambda=850 \mathrm{~nm})$ and converts the macroscopic aspects of the mixtures into two graphics [10]. Acquiring transmission and back scattering data come respectively from the transmission detector which receives the light going across the sample (at $0^{\circ}$ from the incident beam), while the back scattering detector receives the light scattered backward by the sample at $135^{\circ}$ from the incident beam.

\subsection{Samples}

The powder corresponds to a mixture obtained by cogrinding of two micron-size mineral powders (aluminium fluoride and aluminium hydroxide) with the addition of surfactant (polyols) [3] in order to obtain a homogeneous mixture and better flowability of dry powder. Its true density obtained is 2.8 .

The modification of the polarity of the liquid medium is achieved by a variation of the des-ionised water/ethanol ratios (\% weight of ethanol), since the polar parameters of water and alcohol are of 16.8 and 8.8 respectively [15].

It can be noticed that the modifications of liquid composition (density and viscosity of water, ethanol and their mixtures) did not influence much the theoretical particles' velocity of fall as according to the Stokes' law [16]. For example, the velocity of particles with a diameter of twenty microns is $0.39 \mathrm{~cm} \mathrm{~s}^{-1}$ and $0.41 \mathrm{~cm} \mathrm{~s}^{-1}$ in water and ethanol respectively.

For granulometric and electrophoresis measurements, the analysis can only be performed using a very dilute dispersion (about $0.5 \%$ solid weight), whereas the Turbiscan MA2000 analysis requires concentrated dispersions (until $60 \% \mathrm{wt}$ ). In this study, this last concentration of solid has been fixed at the value which corresponds to the industrial concentration of $30 \%$ (wt).

\section{Results and discussion}

\subsection{Laser granulometry and electrophoresis measurements to study agglomeration and dispersion phenomena}

Changes in the particle organisation as a function of alcohol percentage in water were studied by laser diffraction analysis and electrophoresis measurements. In this respect, the water/ alcohol ratios were modified in a range of zero to a hundred percent alcohol.

Particle size distributions in Fig. 1 (for each analysis, dry powder is directly introduce in the analysis cell of granulometer), clearly show both increasing mode values and spreading distribution values as alcohol percentage increases. Fine particles (mode value at $20 \mu \mathrm{m}$ ) are found in water while larger particles (mode value at $50 \mu \mathrm{m}$ ) are observed in ethanol. However, all the intermediate water/alcohol mixtures present quite similar particle size distribution characteristics. So these mineral particles are more dispersed in polar medium in accordance with the principle of compatibility of polarity [1].

Electrophoretic mobility and ionic conductivity were also studied (Fig. 2). Indeed, the Beckman-Coulter Delsa 440 system measure the electrophoretic mobility of particle which related to surface potential (zeta potential) by the Smoluchoowski approximation [8].

These experimental results indicate that dispersion phenomena observed in Fig. 1, is conditioned by greater electrostatic repulsive forces between particles [8], whereas high percentages of alcohol lead to particle attraction. But for suspensions with water/alcohol mixtures, and contrary to the granulometric results, the electrophoretic mobility depends on water/alcohol ratio.

Moreover, in the case of liquid phases containing a majority of water, high values of ionic conductivity $(1.6 \mathrm{mS} / \mathrm{cm})$ indicate dissolution of particles or other chemical reactivity between water and solid mineral phases.

To conclude, in these conditions, the alcohol in liquid phase favors particle agglomeration, whereas in polar media,

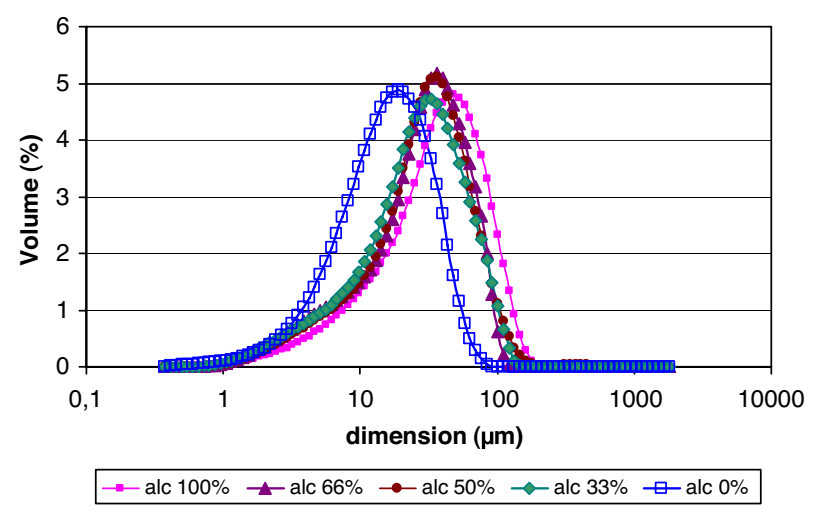

Fig. 1. Evolution of particle size distribution as function of alcohol percentage in the liquid phase. 


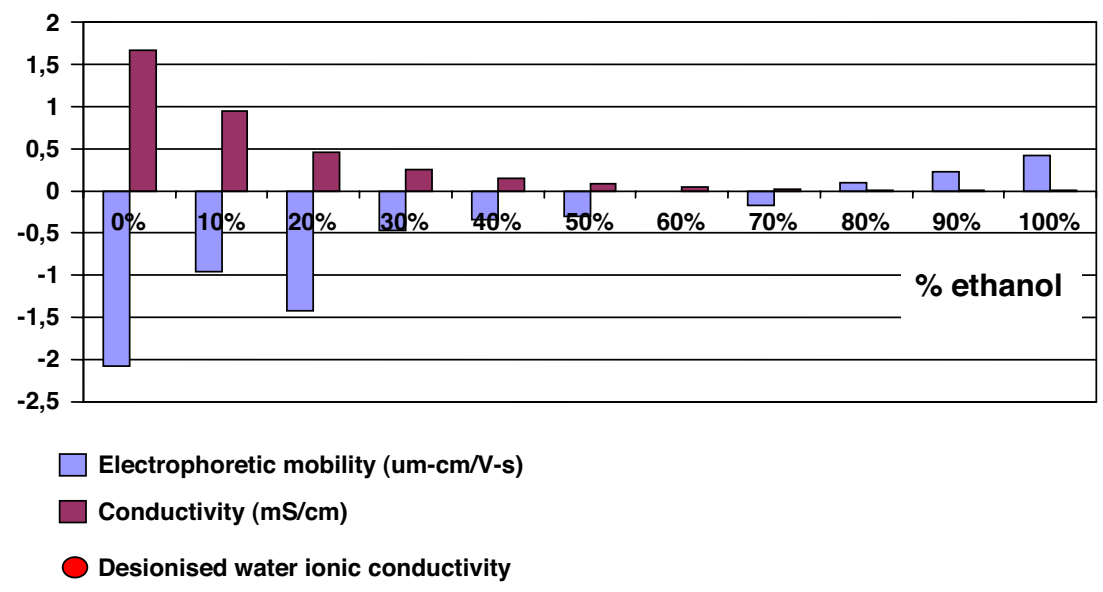

Fig. 2. Electrophoretic mobility and ionic conductivity variations as function of ethanol percentage.

the suspension is electrostatically stabilised. Moreover, in this last case, a chemical reaction between solid and liquid phases may take place. We can point that all alcohol/water mixtures are characterised by the same intermediate state of particle organisation, independently of alcohol rate and variations of electrophoretic mobility values. That is probably due to the hydrodynamic conditions $[17,18]$ of granulometric analysis which involve only one granulometric distribution, until an electrophoretic mobility of approximately $-1 \mu \mathrm{m} \mathrm{cm} / \mathrm{V} . \mathrm{s}$.

\subsection{Turbiscan MA2000 to study the sedimentation behaviour}

The three previous different particle organisations may influence sedimentation behaviour. Therefore the classical clarifying kinetics and the sediment formation were studied in order to find correlations between granular characteristics and sedimentation process. Moreover, the optimum conditions of stability for industrial use could be found.

Before that, visual observations (Fig. 3) show a sedimentation front between a small clear zone without particles at the top of the suspension, and a white opaque sedimentation column. This seems to characterise a compression mode of sedimentation [4] in which particles form a strong enough structure.

Moreover, it was observed that the resuspension of solid seems to become more difficult as the water percentage is

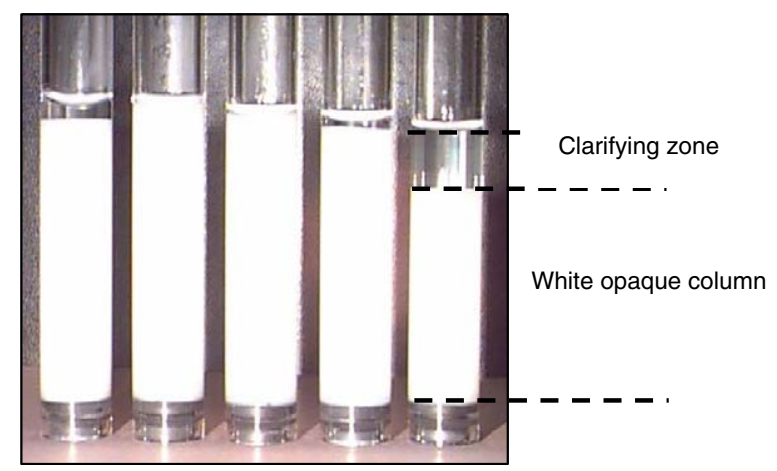

Fig. 3. Sample visual observations. increased. Furthermore, a gelling aspect is observed in aqueous suspension which confirms the chemical reaction suspected in the previous chapter (3.1) but which is not studied in this work. We can however note that the granulometric analysis during only $30 \mathrm{~s}$, this phenomenon will not almost modify the aggregates size measurement.

\subsubsection{Nascent agglomeration phenomena}

The Turbiscan MA2000 optical analyser allows estimating the sedimentation behaviour, in the white opaque column, where the suspension presents a visual homogeneousness. This behaviour is shown by the back scattering profiles versus times, in Fig. 4. Every 5 min a scan is performed along the cylindrical cell during $55 \mathrm{~s}$. Each experiment lasted for $55 \mathrm{~min}$ and includes 12 scans.

These graphics show a very small clarification zone characterised by a small peak at the top of the sample (about $65 \mathrm{~mm}$ of cell height).

The back scattering values present two abrupt variations, at the top and at the bottom of the sample, corresponding respectively to the meniscus (liquid / air interface) and the plug of the cell. Between these two zones, the sedimentation column, for each scan, is homogeneous and presents a constant value of the percentage of back scattering. For this sample (50\% of alcohol) a slight decrease of BS value versus time (of 15.8 to $15.2 \% \mathrm{BS}$ ) was observed (Fig. 4A) and corresponds to a beginning of variation of particle size (agglomeration phenomena) in agreement with following formulas (1) and (2).

Therefore, the middle zone of the profiles between 20 and $40 \mathrm{~mm}$ of cell height, is interesting to study for each suspension. In this zone, the mean value variations of the BS data in function of time are determined for each sample and shown in Fig. 4B. These mean values of BS percentage are approximately constant for each sample but decrease as alcohol percentage increases.

In fact, this variation of the diffuse reflectance values (BS) can be considered to obey the approximate expressions [11]:

$\mathrm{BS} \approx 1 /\left(\lambda^{*}\right)^{1 / 2}$ 


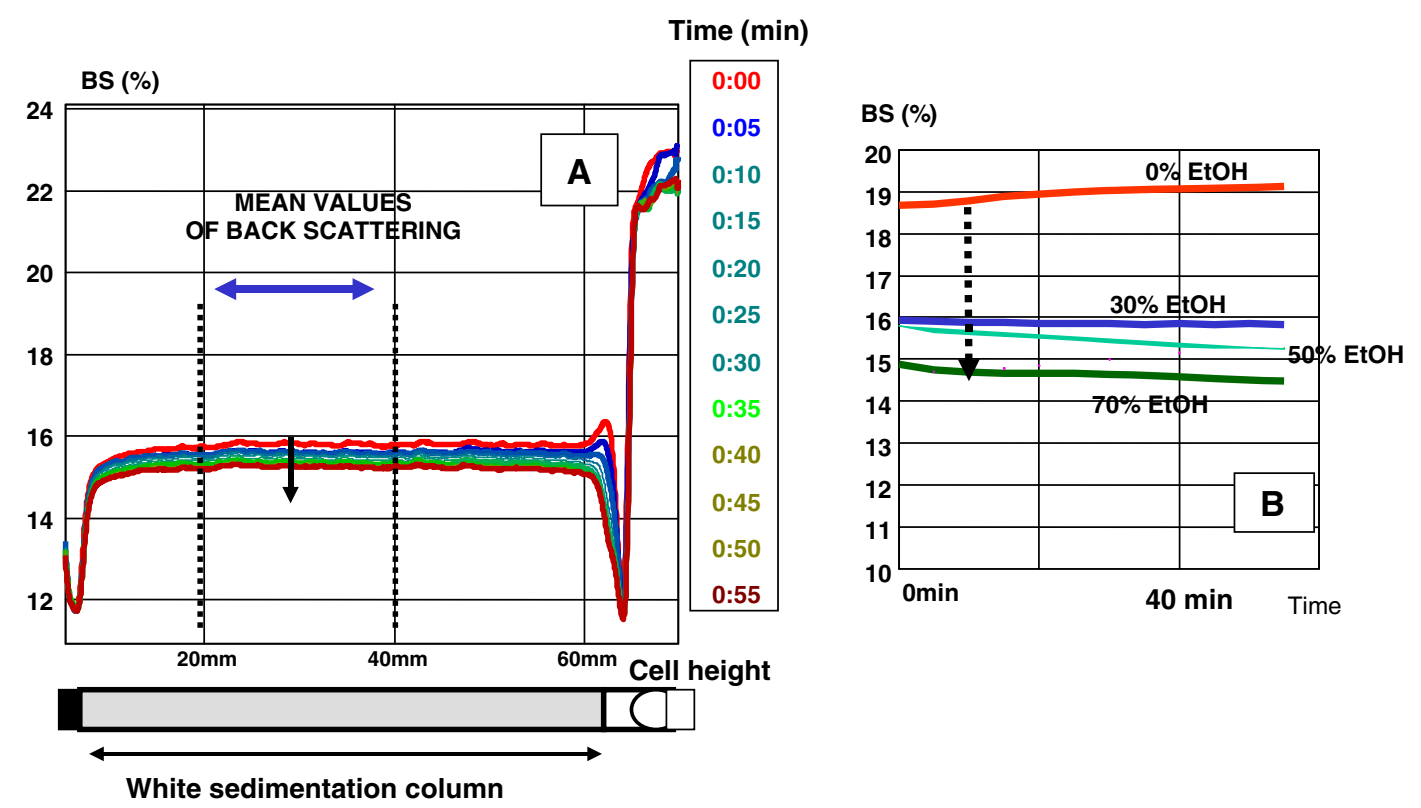

Fig. 4. Evolution of the mean values of back scattering profiles estimate in the middle zone (A) of the column of sedimentation for samples containing 0 to $70 \%$ of ethanol percentage (B).

and

$\lambda^{*}(d, \phi)=2 d /\left[3 \phi(1-g) Q_{\mathrm{s}}\right]$

with, BS (\%) is diffuse reflectance at $135^{\circ}$ detector; $\lambda *(\mu \mathrm{m})$, the photon transport length; $d(\mu \mathrm{m})$, particle diameter; $\phi(\%)$, the particle volume fraction; $g$ and $Q_{\mathrm{s}}$, are two optical parameters in the theory of Lorenz-Mie [12].

In accordance with the Eq. (2), the mean values of BS percentage decrease observed in Fig. $4 \mathrm{~A}$ and $\mathrm{B}$, corresponds to particle size increasing by nascent agglomeration phenomena respectively when the times and ethanol percentage increase. This second variation is in agreement with granulometric results.

\subsubsection{Limit of stabilisation}

Using the same approach, the evolution of the maximum percentage of transmission data corresponding to the scan realised at $55 \mathrm{~min}$ or the relative thickness of this clarifying peak (Fig. 5), studied as function of alcohol percentage (Fig. 6), show a critical quantity of alcohol in water about $60 \%$ wt. (The relative thickness (in \%) corresponds to the fraction of the thickness of the clarifying peak at $5 \%$ of transmission, to the total height of the suspension analysed.)

Above $60 \%$ of alcohol, suspensions present a sedimentation front (Figs. 6B and 7). Below this value, the clarifying zone is insignificant (Figs. 6B and 7).

Then, this critical value of $60 \%$ of ethanol corresponds to the beginning of suspension destabilisation (Fig. 6B) when the polarity of the liquid medium changes. This result is in full agreement with the variation of electrophoretic mobility values which present an "isoelectric point" in this case (Fig. 2).

In order to quantify this destabilisation of the suspensions, observed at the top of the sample, the absolute thickness versus elapsed time was measured. From these results, the clarifying kinetics could be calculated (Fig. 7). It corresponds to the slope (in $\mathrm{mm} / \mathrm{min}$ ) of the curve of the evolution of absolute thickness of the clarifying peak (at $5 \%$ of transmission) versus elapsed times.

For suspensions presenting a sedimentation front, clarifying speed values vary from 0.14 to $0.2 \mathrm{~mm} / \mathrm{min}$. On the other hand, it is difficult to determine the clarifying speed when the suspension is stabilised because of the thinness of the clarifying zone.

To summarise, a limit of stabilisation can be determined as well by the evolution of maximum percentage of transmission as relative thickness of the clarifying zone and quantified by clarifying speed. It corresponds to an "isoelectric point" [8] identified by electrophoresis measurement.

\subsubsection{Two destabilisation behaviours}

At the opposite of the clarifying zone, the bottom zone of all the samples was studied by acquiring back scattering data.

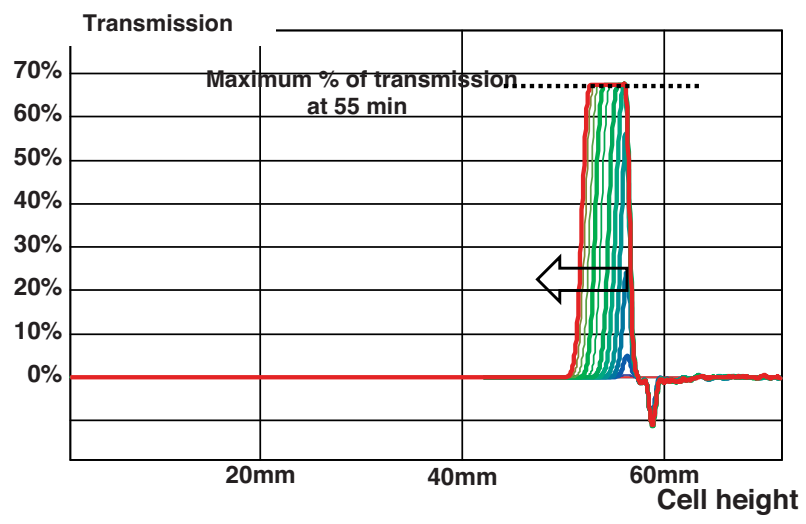

Fig. 5. Study of the transmission data in the clarifying zone for a sample containing $80 \%$ of alcohol percentage. 

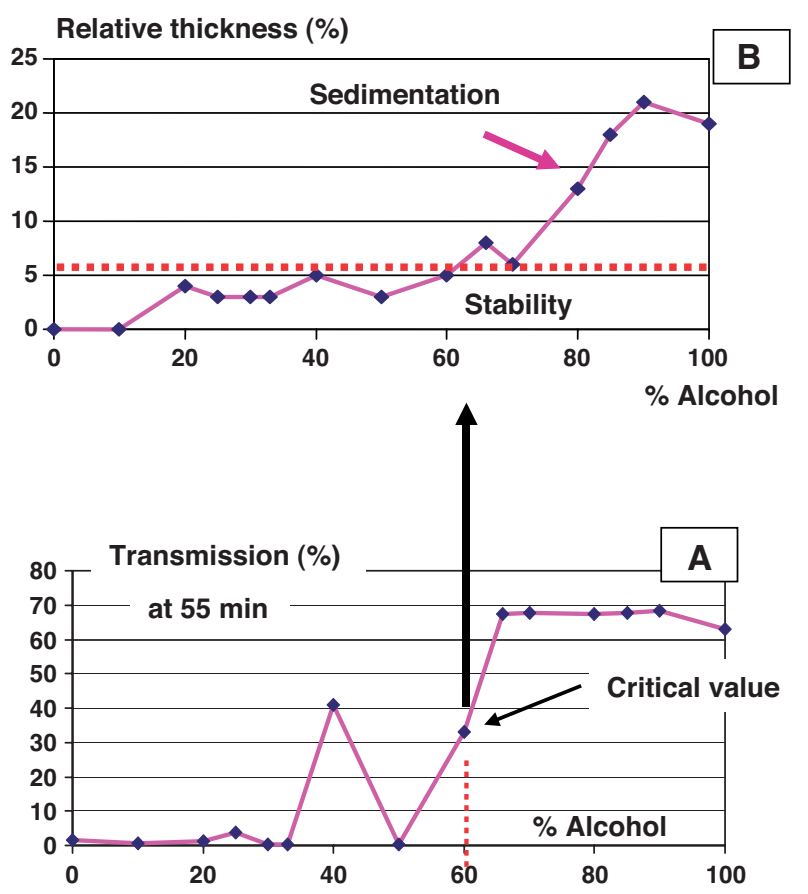

Fig. 6. Maximum percentage of transmission (A) and relative thickness (B) of clarifying zone, at $55 \mathrm{~min}$, versus alcohol percentage.

As shown previously, lowest alcohol ratios lead to homogeneous sedimentation column, and a back scattering level increasing with time (Fig. 8A). This effect indicates a decreasing particle size with time (Eq. (2)). Below 33\% wt alcohol, this last phenomenon is less important as the alcohol ratio increases. Consequently, there is no sediment formation and particles dispersed.

For 33\% wt of alcohol, the sedimentation column is still homogeneous and the back scattering level is stable versus times (Fig. 8B). But from this value and in contrast with the preceding samples, this level decreased according with time and proportionally to alcohol quantity (Fig. 8C). This result indicates a nascent agglomeration state which occurs before the limit of stability identified in the previous chapter. The electrostatic repulsion (Fig. 2) becomes insufficient to stabilise the suspension, but there is no particle migration within the sedimentation column.

When alcohol percentage is about $70 \%$, a crossing of curves arises in the bottom zone due to the beginning of particle migration (Fig. 8D). Indeed, the back scattering signal represents two phenomena, the variation of " $\phi$ " and simultaneous variation of " $d$ " versus times, which increases as ethanol ratio increases. This leads to compact on of the column. A large volume of sediment, about $60 \%$ of the total suspension height is formed (Fig. 8E).

Therefore, nascent agglomeration phenomena have been shown by the study of sediment formation, whereas it has not been possible by electrophoresis measurements or study of clarifying kinetics.

These results permit to fix at $33 \%$ wt of alcohol, the choice of industrial liquid mixture to obtain a maximum stability while minimizing the chemical reactivity of solid phase with liquid.

\section{Conclusion}

A better understanding of sedimentation behaviours of mineral suspensions has been investigated by three complementary optical methods of dispersed systems analysis: laser granulometry, electrophoresis measurements, and concentrated dispersion analyser. Although these three techniques require very different concentrations of solid (respectively 0.5 and $30 \%$ solid weight), and different hydrodynamic conditions, the results obtained are in good agreement.

Laser granulometry and electrophoresis measurements clearly show that the dispersion of particles is due to two combined phenomena: a high value of surface potential which

\section{Clarifying speed $(\mathrm{mm} / \mathrm{min})$}

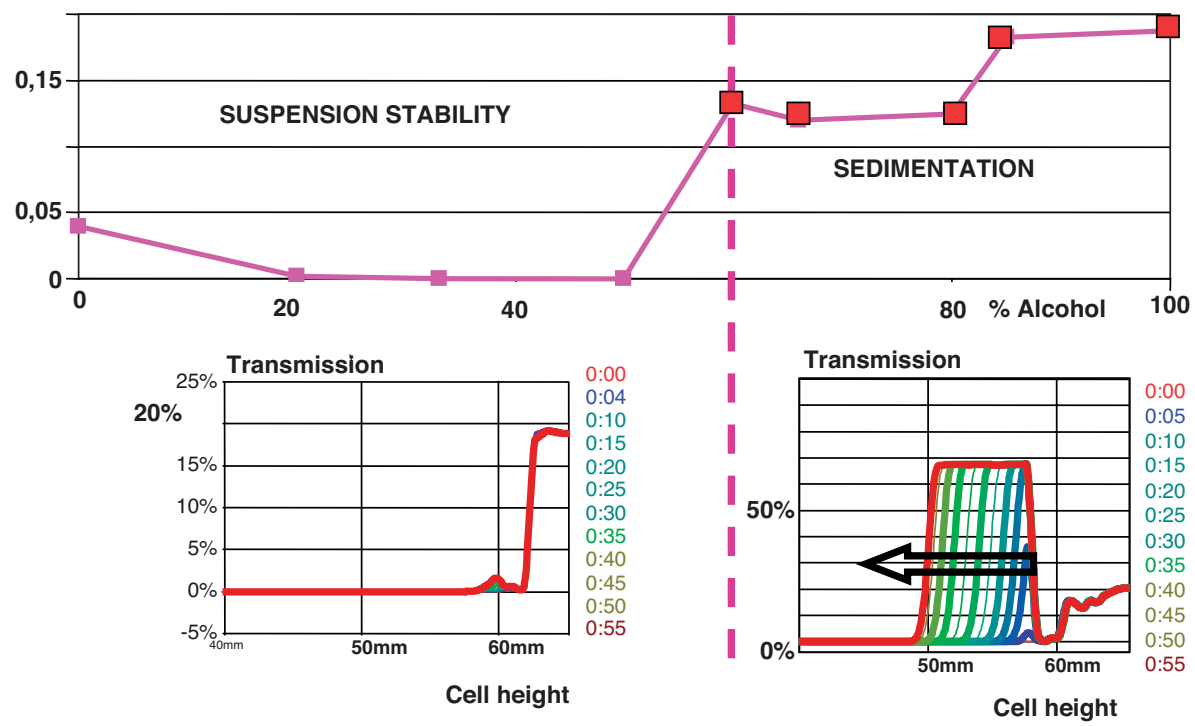

Fig. 7. Clarifying speed according to alcohol percentage. 
- alcohol $0 \%$

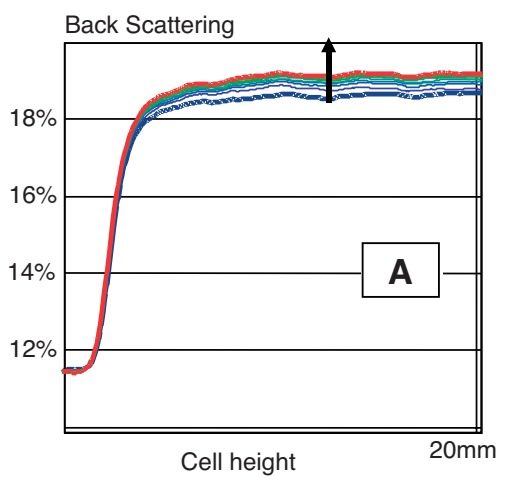

- alcohol $50 \%$

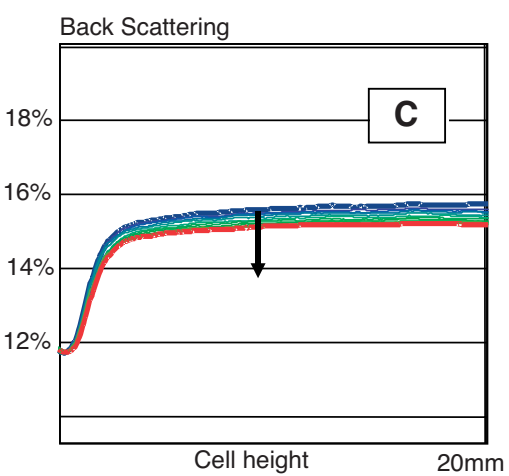

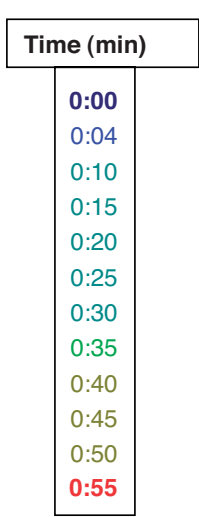

- alcohol $33 \%$

Back Scattering

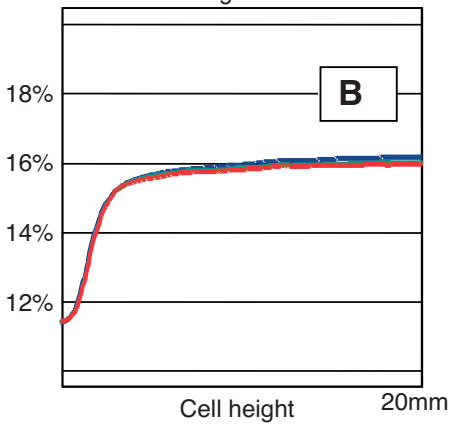

-alcohol $70 \%$

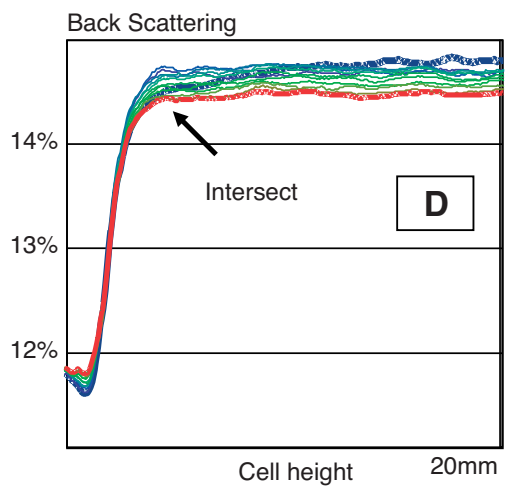

-alcohol $100 \%$

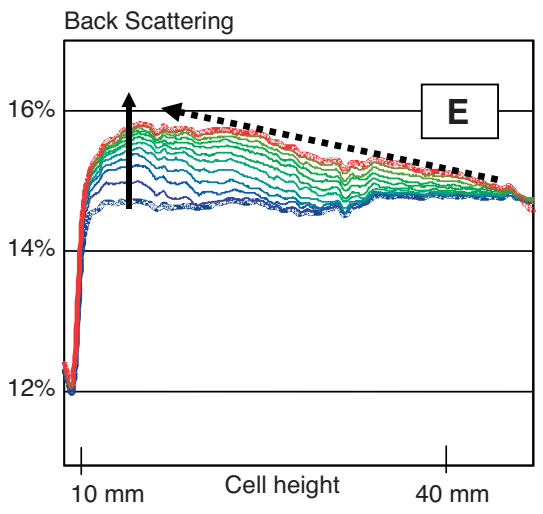

Fig. 8. Particle size variation and sediment formation in the sedimentation column according to alcohol percentage.

stabilises the suspension by electrostatic repulsive interaction, and also, chemical reactivity of mineral particles with water leading to a gelling aspect.

Furthermore, a limit of suspension stability has been identified by electrophoretic measurements and the study of clarification of suspension by the dispersion analyser. In this last case, this limit of stability can be estimated by the clarifying speed measurements, the values of relative thickness or maximum percentage of the clarifying peak in transmission profiles.

However, only the study of sediment formation by Turbiscan MA 2000 has shown that before this limit of stabilisation, nascent agglomeration phenomena occurs although the suspension seems globally stabilised. In addition, by this last approach, two destabilisation behaviours have been identified: one corresponding to the global migration of particles without sediment formation which corresponds to compression settling. The other corresponds to sediment formation due to migration of agglomerates down the column.

In this work, the complementary use of these three optical methods seems to be necessary to investigate sedimentation behaviours. However, the study of sediment formation seems to be the most relevant parameter which clearly identifies the beginning of destabilisation. These results can be extended to characterise all type of suspension.

\section{References}

[1] Jun Ren, Wenmei Wang, Shouci Lu, Jian Shen, Fangqiong Tang, Characteristics of dispersion behaviour of fine particles in different liquid media, Powder Technology 137 (2003) 91-94. 
[2] V.M. Gun'ko, V.I. Zarko, R. Leboda, E. Chibowski, Aqueous suspension of fumed oxides: particle size distribution and zeta potential, Advances in Colloid and Interface Science 91 (1) (2001) 1-112.

[3] A. Kouadri-Henni, N. Azema, A. Benhassaine, Flowability of a mixture of powders obtained by co-grinding, mixing and surface treatment, Powder Technology 103 (1999) 37-43.

[4] P. Coussot, C. Ancey, Rhéophysique des Pâtes et des Suspensions, EDP Sciences, 1999.

[5] B. Fitch, Sedimentation of flocculent suspensions: state of the art, The American Institute of Chemical Engineers Journal 25 (6) (1979) 913-930.

[6] Hideto Yoshida, Tantular Nurtono, Kunihiro Fukui, A new method for control of dilute suspension sedimentation by horizontal movement, Powder Technology 150 (31) (2005) 9-19.

[7] P. Garrido, F. Concha, R. Bürger, Setting velocities of particulate systems: 14. Unified model of sedimentation, centrifugation and filtration of flocculated suspensions, International Journal of Mineral Processing 72 (1-4) (2003) 57-74.

[8] R.J. Hunter, Zeta Potential in Colloid Science, Academic Press, Inc., 1986 second printing.

[9] T. Allen, Particle Size Measurement, Ed. Chapman and Hall, London (5th Ed., 1997).

[10] G. Meunier, Le TURBISCAN: un nouvel instrument de mesure de phénomènes naissants de démixtion dans les émulsions et les suspensions, Spectra Analyse 179 (1994) 53-58.
[11] O. Mengual, G. Meunier, I. Cayre, K. Puech, P. Snabre, Turbiscan MA2000: multiple light scattering measurement for concentrated emulsion and suspension instability analysis, Talanta 50 (2) (1999) 445-456.

[12] O. Characterisation of instability of concentrated dispersions by a new optical analyser : the Turbiscan MA 1000, Colloids and surfaces - A: Physicochemical and Engineering Aspects 152 (1999) 111-123.

[13] M. Heuer, K. Leschonski, Results obtained with a new instrument for the measurement of particle size distributions from diffraction patterns, Particle Characterization 2 (1985) 7-13.

[14] J.K. Harfield, R.C. Bunker, Zeta Potential and Electrophoretic Mobility Measurement by Laser Doppler Velocimetry, Corporate Ref. Lab., Coulter Electronics Ltd., Luton, Presented at INPARTEC, Imperial Coll., London, September 13th-14th (1988), 11pp.

[15] C.M. Hansen, Hansen Solubility Parameters - A User's Handbook, CRC Press, LLC, 2000.

[16] P. Tang, J.A Raper, Modelling the settling behaviour of fractal aggregates - a review, Powder Technology 123 (2-3) (2002) 114-125.

[17] C.A. Biggs, P.A. Lant, Modelling activated sludge flocculation using population balances, Powder Technology 124 (3) (2002) 201-211.

[18] C. Selomulya, G. Bushell, R. Amal, T.D Waite, Aggregate properties in relation to aggregation conditions under various applied shear environments, Industrial Journal of Mineral Processing, Vol. 73, 2-4,3 (2004) 295-307. 